\title{
Transformational Teamwork: The Virtues and Values of Collaboration
}

Decades of innovation and technological advances have led to progress in biomedical sciences, medicine, and public health. However, today's complex health systems exhibit inconsistent health care quality, escalating costs, inequities in access, and shortcomings in improvement in population health outcomes ${ }^{1}$. The increased complexity of health care systems necessitates an increasing emphasis on teamwork; this requires dynamic partnerships with research networks, as well as clinical and delivery system innovations, to improve health care value continually. Efforts by health professionals to improve patient care and population health have been challenging, in spite of the collaborative focus of the health profession.

Team practice is common in heath care services, health research, health education and health policy. There are several different approaches to team practice, each with differing attributes and functions. $^{2}$ One is a multidisciplinary practice which utilizes the knowledge and experiences of individuals from diverse disciplines, with each discipline approaching the tasks from its own perspective. Essentially, health professionals work in conjunction with each other but act autonomously. Second, interdisciplinary practice expands the multidisciplinary team through collaborative communication and interdependent practice. This is a more integrated approach as members build upon other members' expertise. Third is the transdisciplinary practice approach, where the team members are more flexible with their roles and responsibilities. The transdisciplinary team members share knowledge, skills, and responsibilities across traditional discipline boundaries in assessment, diagnosis, planning and implementation.

Central to the success of any approach to team practice in health care systems are the core values of caring ${ }^{4}$ that team members demonstrate to become high functioning teams and to increase team effectiveness. ${ }^{5,6,7,8,9,10,11}$

Honesty: Honesty is critical for maintaining the mutual trust necessary for a high-functioning team. Communication including transparency about aims, decisions, uncertainty, and mistakes within the team is essential.

Discipline: Team member discipline is critical not only to carry out their roles and responsibilities towards the shared goal but also to seek out and share new information and improve individual and team functioning. Such discipline allows teams to stay focused and abide by their processes and protocol as they find ways to improve.

Humility: Team members recognize and acknowledge differences in training and understand that fellow team members can rely on each other to help identify and avert failures, regardless of where they are in the hierarchy. Team members demonstrate confidence without arrogance while working with members of other health professions.

Readiness for Collaboration: This willingness comes in the form of one's adaptability, flexibility, and openness to diverse perspectives, communication skills, conflict resolution, and respect for others. Each of these qualities is essential for collaboration and group productivity. 
Curiosity: Team members follow reflective practice upon the lessons learned from their activities. They draw upon their own insights for continuous quality improvement of their individual work and the functioning of the team.

In conclusion, teams with underlying core values hold the potential to transform the health care system. However, to capture the full potential of high-functioning teams, institutions, health care organizations, governments, and insurance industry must invest in the people and processes that lead to improved outcomes ${ }^{12}$. From this empowered foundation, new heights can be reached through teamwork.

\section{References}

1. Health professions education: A bridge to quality, 2003, Institute of Medicine. The National Academies Press

2. Choi, Bernard CK, Park, Anita WP. Multidisciplinarity, interdisciplinarity, and transdisciplinarity in health research, services, education and policy: 2. Promotors, barriers, and strategies of enhancement. 2007, Clinical and Investigative Medicine.

3. Establishing Transdisciplinary Professionalism for Improving Health Outcomes: Workshop Summary. Institute of Medicine. The National Academies Press

4. Core Principles \& Values of Effective Team-Based Health Care. Pamela Mitchell, Matthew Wynia, et. al.. 2012 Institute of Medicine. The National Academies Press.

5. The Wisdom of Teams: Creating the High-Performance Organization. Jon R. Katzenbach and Douglas K. Smith

6. The Discipline of Teams: Jon R. Katzenbach and Douglas K. Smith. 2005 Harvard Business Review

7. Hall, Kara L. et al. "The Collaboration Readiness of Transdisciplinary Research Teams and Centers: Findings from the National Cancer Institute's TREC Year-One Evaluation Study." American Journal of Preventive Medicine 35.2 Suppl (2008): S161-S172. PMC. Web. 27 July 2016.

8. The Five Dysfunctions of a Team: Team Assessment. Patrick Lencioni

9. Enhancing The Effectiveness of Team Science, 2015, National Academies Press.

10. Martine Haas \& Mark Mortensen. The Secrets of Great Teamwork, 2016., Harvard Business Review.

11. The hard science of teamwork by Alex"Sandy"Pentland March 2012 Harvard Business Review.

12. The future of nursing: Leading change, advancing health: 2011 Institute of Medicine. The National Academies Press. 